\title{
New applications of Schrödinger type inequalities in the Schrödingerean Hardy space
}

\author{
Yong Lu' ${ }^{1}$, Liang Kou², Jianguo Sun²*, Guodong Zhao², Wenshan Wang ${ }^{2}$ and Qilong Han²
}

\section{"Correspondence: \\ jgsunhar@foxmail.com \\ ${ }^{2}$ Department of Computer Science \\ and Technology, Harbin \\ Engineering University, Harbin, \\ 150001, China \\ Full list of author information is \\ available at the end of the article}

\begin{abstract}
As new applications of Schrödinger type inequalities obtained by Jiang (J. Inequal. Appl. 2016: Article ID 247, 2016) in the Schrödingerean Hardy space, we not only obtain the representation of Schrödingerean harmonic functions but also give a sufficient and necessary condition between the Schrödingerean distributional function and its derivative in the Schrödingerean Hardy space.
\end{abstract}

Keywords: Schrödinger type inequality; Schrödingerean Hardy space; upper half-plane

\section{Introduction}

The Schrödingerean Hardy spaces $H^{p}\left(\mathbb{C}_{+}\right)(1<p<\infty)$ are defined to consist of those functions $f$, Schrödingerean holomorphic in the upper half-plane $\mathbb{C}_{+}=\{z=x+i y: y>0\}$ with the property that $M_{p}(f, y)$ is uniformly bounded for $y>0$, where

$$
M_{p}(f, y)=\left(\int_{-\infty}^{+\infty}|f(x+i y)|^{p} d x\right)^{\frac{1}{p}}
$$

Since $|f|^{p}$ is Schrödingerean subharmonic for $f \in H^{p}\left(\mathbb{C}_{+}\right)$with respect to $S c h_{a}$, the function $M_{p}(f, y)$ decreases in $(0, \infty)$,

$$
\|f\|_{H^{p}\left(\mathbb{C}_{+}\right)}=\sup \left\{M_{p}(f, y): 0<y<\infty\right\}=\lim _{y \rightarrow 0} M_{p}(f, y) .
$$

If $f(x)$ is the non-tangential boundary limits of the Schrödingerean function $f \in H^{p}\left(\mathbb{C}_{+}\right)$, then $f(x) \in L^{p}(\mathbb{R})$ and

$$
\|f\|_{p}=\left(\int_{-\infty}^{+\infty}|f(x)|^{p} d x\right)^{\frac{1}{p}}=\|f\|_{H^{p}\left(\mathbb{C}_{+}\right)} .
$$

A function $\phi(t)$ defined on $\mathbb{R}$ belongs to the space $\mathcal{D}_{L^{p}}, 1<p<\infty$, iff

(1) $\phi(t) \in \mathcal{C}^{\infty}$;

(2) $\phi^{(k)}(t) \in L^{p}$ for all $k \in \mathbb{N}$, where $\mathbb{N}$ is the set of nonnegative integers. 
The space $\mathcal{D}$ consists of infinitely differentiable complex-valued functions defined on $\mathbb{R}$. In the sequel, for $1<p<\infty$, we will write

$$
p^{\prime}=\frac{p}{p-1}
$$

and denote by $\mathcal{D}_{L^{p}}^{\prime}$ the dual of the space $\mathcal{D}_{L^{p^{\prime}}}$, that is, $\mathcal{D}_{L^{p}}=\left(\mathcal{D}_{L^{p^{\prime}}}\right)^{\prime}$. We also denote by $D^{\prime}$ the dual of the space $D$. So we can get $D \subseteq \mathcal{D}_{L^{p}}$ and $\mathcal{D}_{L^{p}}^{\prime} \subseteq D^{\prime}$.

Definition 1.1 (see [2]) Let $f \in \mathcal{D}^{\prime}$. An analytic representation of $f$ is any function $F(z)$ defined and analytic on the complement of the support of $f$ such that for all test functions $\phi \in \mathcal{D}$,

$$
\lim _{y \rightarrow 0^{+}} \int_{-\infty}^{+\infty}[F(x+i y)-F(x-i y)] \phi(x) d x=\langle f(x), \phi(x)\rangle .
$$

Definition 1.2 Let $f \in \mathcal{D}_{L^{p}}^{\prime}(1<p<\infty)$ and assume that $D f$ is the operator of distributional differentiation defined on $\mathcal{D}_{L^{p}}^{\prime}$ by

$$
\langle D f, \phi\rangle=\langle f,-D \phi\rangle
$$

for all $\phi \in \mathcal{D}_{L^{p^{\prime}}}$.

Then

$$
D f \in \mathcal{D}_{L^{p}}^{\prime}
$$

Since $f \in \mathcal{D}_{L^{p}}^{\prime}, D \varphi \in D_{L^{p^{\prime}}}, D f$ defined as above is a functional on $D_{L^{p^{\prime}}}$. Linearity of $D f$ is trivial. Assume that $\left\{\varphi_{v}\right\} \rightarrow \varphi$ in $D_{L^{p^{\prime}}}$. Then

$$
\left\langle D f, \varphi_{v}\right\rangle=\left\langle f,-D \varphi_{\nu}\right\rangle \rightarrow\langle f,-D \varphi\rangle=\langle D f, \varphi\rangle .
$$

\section{Main results}

In 2016, Jiang (see [1]) proved Schrödinger type inequalities for stabilization of discrete linear systems associated with the stationary Schrödinger operator. As applications, Jiang and Uso (see [3]) obtained boundary behaviors for linear systems of subsolutions of the stationary Schrödinger equation. Almost at the same time, Huang (see [4]) considered a new type of minimal thinness with respect to the stationary Schrödinger operator. As an application, Huang and Ychussie (see [5]) solved the Dirichlet-Sch problems on smooth cones with slow-growth continuous data. Recently, Lü and Ülker (see [6]) gave the existence of weak solutions for two-point boundary value problems of the Schrödingerean predator-prey system. Motivated by their results, by using Schrödinger type inequalities proved by Jiang (see [1]), we obtain the integral representation of Schrödingerean harmonic functions in the Schrödingerean Hardy space.

Theorem 2.1 Suppose that $1<p<\infty$ and $f \in \mathcal{D}_{L^{p}}^{\prime}$. Then

$$
F(Z)=\frac{1}{2 \pi i}\left\langle f(t), \frac{1}{t-z}\right\rangle
$$


is one of the analytic representations of $f$, which satisfies

$$
\sup _{-\infty<x<\infty, y \geq \delta>0}\|F(x+i y)\|=A_{\delta}<\infty
$$

and

$$
\sup _{-\infty<x<\infty}\|F(x+i y)\|=O\left(y^{-\frac{1}{p}}\right)
$$

where $y \rightarrow \infty$.

There exist functions $F_{k}(z) \in H^{p}\left(\mathbb{C}_{+}\right)$, so that

$$
F(z)=\sum_{k=1}^{r} \frac{\partial^{k-1}}{\partial z^{k-1}} F_{k}(z)
$$

and

$$
F^{(j)}(z)=\sum_{k=1}^{r} \frac{\partial^{k+j-1}}{\partial z^{k+j-1}} F_{k}(z),
$$

where $r$ and $j$ are nonnegative integers.

Theorem 2.2 If $1<p<\infty, F_{k} \in H^{p}\left(\mathbb{C}_{+}\right)$and

$$
F(z)=\sum_{k=1}^{r} \frac{\partial^{k-1}}{\partial z^{k-1}} F_{k}(z)
$$

then there exists a distributional function $f(x) \in \mathcal{D}_{L^{p}}^{\prime}$ such that $F(z)$ is one of analytic representations of $f(x)$.

Corollary 2.3 If $1<p<\infty$ and $f(x) \in \mathcal{D}_{L^{p}}^{\prime}$, then

$$
F(Z)=\frac{1}{2 \pi i}\left\langle f(t), \frac{1}{t-z}\right\rangle
$$

satisfies

$$
\sup _{-\infty<x<\infty, y \geq \delta>0}\|F(x+i y)\|=A_{\delta}<\infty
$$

and

$$
\sup _{-\infty<x<\infty}\|F(x+i y)\|=O\left(y^{-\frac{1}{p}}\right),
$$

where $y \rightarrow \infty$.

There exist functions $F_{k}(z)$ in $H^{p}\left(\mathbb{C}_{+}\right)$so that

$$
F(z)=\sum_{k=1}^{r} \frac{\partial^{k+j-1}}{\partial z^{k+j-1}} F_{k}(z)
$$

where $j>1, r, j$ are nonnegative integers. 


\section{Lemmas}

In this section we need the following lemmas.

Lemma 3.1 (see $[7,8])$ If $1<p<\infty, u(t) \in L^{p}(\mathbb{R})$ and the function $G(u)(t)$ is defined as follows

$$
G(u)(t)=\frac{1}{2 \pi i} \int_{-\infty}^{\infty} \frac{u(t)}{t-z} d t
$$

then

$$
G(u)(t) \in H^{p}\left(\mathbb{C}_{+}\right) .
$$

Lemma 3.2 (see [1]) Let $F(z)$ be an analytic complex-valued function of the complex variable $z=x+$ iy in the open upper half-plane satisfying

(1) for fixed $y>0, p^{\prime}=\frac{p}{p+1}, 1<p<\infty, F(x+i y) \in L^{p}$;

(2)

$$
\lim _{y \rightarrow 0^{+}} F(x+i y)=f^{+}(x)
$$

in $\mathcal{D}_{L^{p}}^{\prime}$ (weakly)

$$
\sup _{-\infty<x<\infty}\|F(x+i y)\| \rightarrow O,
$$

where $y \rightarrow \infty$ and

$$
\sup _{-\infty<x<\infty, y \geq \delta>0}\|F(x+i y)\|=A_{\delta}<\infty .
$$

Then

$$
F(z)=\frac{1}{2 \pi i}\left\langle f^{+}(t), \frac{1}{t-z}\right\rangle,
$$

where $\operatorname{Im} z>0$.

\section{Proofs of the main results}

\subsection{Proof of Theorem 2.1}

In view of the structure formula in [3], for $f \in \mathcal{D}_{L^{p}}^{\prime}$,

$$
F(z)=\frac{1}{2 \pi i}\left\langle f^{+}(t), \frac{1}{t-z}\right\rangle,
$$

there exists a nonnegative integer $r$ and $f_{k} \in L_{p}$ such that

$$
\begin{aligned}
F(z) & =\frac{1}{2 \pi i} \sum_{k=1}^{r} \int_{\mathbb{R}} f_{k}(t)\left(-\frac{\partial}{\partial t}\right)^{(k-1)}\left(\frac{1}{t-z}\right) d t \\
& =\frac{1}{2 \pi i} \sum_{k=1}^{r} \int_{\mathbb{R}} f_{k}(t)(-1)^{k-1} \frac{(k-1) !}{(t-z)^{k}} d t .
\end{aligned}
$$


So

$$
|F(x+i y)| \leq \frac{1}{2 \pi} \sum_{k=1}^{r} \int_{\mathbb{R}}\left|f_{k}(t)\right| \frac{(k-1) !}{|t-z|^{k}} d t
$$

By using Holder's inequality, we have

$$
|F(x+i y)| \leq \frac{1}{2 \pi} \sum_{k=1}^{r}(k-1) !\left(\int_{\mathbb{R}}\left|f_{k}(t)\right|^{p} d t\right)^{\frac{1}{p}}\left(\int_{R} \frac{1}{|t-z|^{k p^{\prime}}} d t\right)^{\frac{1}{p^{\prime}}}
$$

Define

$$
I=\int_{R} \frac{1}{|t-z|^{k p^{\prime}}} d t
$$

So

$$
\begin{aligned}
I & =\int_{\mathbb{R}} \frac{1}{\left[(t-x)^{2}+y^{2}\right]^{\frac{k p^{\prime}}{2}}} d t \\
& =\int_{\mathbb{R}} \frac{1}{y^{k p^{\prime}}\left[\left(\frac{t}{y}\right)^{2}+1\right]^{\frac{k p^{\prime}}{2}}} d t \\
& =\frac{1}{y^{k p \prime-1}} \int_{\mathbb{R}} \frac{1}{\left(1+t^{2}\right)^{\frac{k p^{\prime}}{y}}} d t .
\end{aligned}
$$

Further, $k>1$ and $p^{\prime}>1$ imply that $k p^{\prime}>1$ for $y \geq \delta>0$, there exists a constant $C$ satisfying

$$
I \leq \frac{C}{\delta^{k p \prime-1}}<\infty
$$

Since $f_{k} \in L^{p}$, there exists a constant $M$ such that

$$
\sup _{-\infty<x<\infty, y \geq \delta>0}\|F(x+i y)\|=A_{\delta}<\infty,
$$

where

$$
\begin{aligned}
& A_{\delta}=\frac{1}{2 \pi} \sum_{k=1}^{r}(k-1) ! \frac{M C^{\frac{1}{p^{\prime}}}}{\frac{k \prime-1}{\delta p^{\prime}}}, \\
& \left|y^{\frac{1}{p}} F(x+y i)\right| \leq \sum_{k=1}^{r}(k-1) !\left\|f_{k}\right\|_{L}^{p} \frac{1}{y^{k p \prime-1-\frac{1}{p}}} \int_{\mathbb{R}} \frac{1}{\left(1+t^{2}\right)^{\frac{k p^{\prime}}{y}}} d t
\end{aligned}
$$

and

$$
k p^{\prime}-1-\frac{1}{p}=p^{2}(1-k)-1<0 .
$$

So

$$
\lim _{y \rightarrow \infty} \sup _{-\infty<x<\infty}|F(x+y i)|=O\left(y^{-\frac{1}{p}}\right) .
$$


In view of the structure formula (see [9])

$$
\begin{aligned}
F(z) & =\frac{1}{2 \pi i} \sum_{k=1}^{r} \int_{\mathbb{R}} f_{k}(t)\left(-\frac{\partial}{\partial t}\right)^{(k-1)}\left(\frac{1}{t-z}\right) d t \\
& =\frac{1}{2 \pi i} \sum_{k=1}^{r} \int_{\mathbb{R}} f_{k}(t)\left(\frac{\partial}{\partial z}\right)^{(k-1)}\left(\frac{1}{t-z}\right) d t \\
& =\sum_{k=1}^{r}\left(\frac{\partial}{\partial z}\right)^{(k-1)} \frac{1}{2 \pi i} \int_{\mathbb{R}} \frac{f_{k}(t)}{t-z} d t \\
& =\sum_{k=1}^{r}\left(\frac{\partial}{\partial z}\right)^{(k-1)} F_{k}(z),
\end{aligned}
$$

where

$$
F_{k}(z)=\frac{1}{2 \pi i} \int_{\mathbb{R}} \frac{f_{k}(t)}{t-z} d t
$$

According to Lemma 3.1, we know that $F_{k}(z) \in H^{p}\left(C_{+}\right)$, which gives that

$$
F^{(j)}(z)=\sum_{k=1}^{r}\left(\frac{\partial}{\partial z}\right)^{(k+j-1)} F_{k}(z)
$$

where $j$ is a nonnegative integer.

\subsection{Proof of Theorem 2.2}

Since $F_{k}(z) \in H^{p}\left(C_{+}\right)$, there exist functions $f_{k}(t) \in L^{p}$, where $f_{k}$ is the non-tangential limit of $F(z)$, where $F_{k}(x+i y) \in L^{p}$ for fixed $y$.

Since $D_{L^{p^{\prime}}} \in L^{p^{\prime}}$, we see that $f_{k}(t) \in D_{L^{p}}^{\prime}$. By using the property of a subharmonic function, we get

$$
\begin{aligned}
\left|F_{k}(x+i y)\right|^{p} & \leq \frac{1}{\pi y^{2}} \int_{D(x+i y, y)}\left|F_{k}(\xi+i \eta)\right|^{p} d \lambda \\
& \leq \frac{1}{\pi y^{2}} \int_{x-y}^{x+y} \int_{0}^{2 y}\left|F_{k}(\xi+i \eta)\right|^{p} d \eta d \zeta \\
& \leq \frac{2}{\pi y}\left\|F_{k}\right\|_{H^{p}}^{p} .
\end{aligned}
$$

So

$$
\left|F_{k}(x+i y)\right| \leq\left(\frac{2}{\pi y}\left\|F_{k}\right\|_{H^{p}}^{p}\right)^{\frac{1}{p}}=y^{\frac{1}{p}}\left(\frac{2}{\pi}\left\|F_{k}\right\|_{H^{p}}^{p}\right)^{\frac{1}{p}}
$$

which gives that

$$
F_{k}(x+i y)=O\left(\frac{1}{y \frac{1}{p}}\right)
$$


where $y>0$ and

$$
\sup _{-\infty<x<\infty, y \geq \delta>0}\|F(x+i y)\| \leq \frac{2}{\pi \delta}\left\|F_{k}\right\|_{H^{p}}^{p}=A_{\delta}<\infty .
$$

According to Lemma 3.2, we know that $F_{k}(z)$ can be written as

$$
F_{k}(x)=\frac{1}{2 \pi i}\left\langle f_{k}(t), \frac{1}{t-z}\right\rangle \text {. }
$$

So

$$
\begin{aligned}
F(z) & =\sum_{k=1}^{r}\left(\frac{\partial}{\partial z}\right)^{k-1} F_{k}(z) \\
& =\sum_{k=1}^{r}\left(\frac{\partial}{\partial z}\right)^{k-1} \frac{1}{2 \pi i}\left\langle f_{k}(t), \frac{1}{t-z}\right\rangle \\
& =\frac{1}{2 \pi i} \sum_{k=1}^{r}\left\langle f_{k}(t),\left(\frac{\partial}{\partial z}\right)^{k-1} \frac{1}{t-z}\right\rangle \\
& =\frac{1}{2 \pi i} \sum_{k=1}^{r}\left\langle D^{(k-1)} f_{k}(t), \frac{1}{t-z}\right\rangle \\
& =\frac{1}{2 \pi i}\left\langle\sum_{k=1}^{r} D^{(k-1)} f_{k}(t), \frac{1}{t-z}\right\rangle,
\end{aligned}
$$

which gives that

$$
\begin{aligned}
F(z) & =\sum_{k=1}^{r}\left(\frac{\partial}{\partial z}\right)^{k-1} F_{k}(z) \\
& =\sum_{k=1}^{r}\left(\frac{\partial}{\partial z}\right)^{k-1} \frac{1}{2 \pi i}\left\langle f_{k}(t), \frac{1}{t-z}\right\rangle \\
& =\frac{1}{2 \pi i} \sum_{k=1}^{r}\left\langle f_{k}(t),\left(\frac{\partial}{\partial z}\right)^{k-1} \frac{1}{t-z}\right\rangle \\
& =\frac{1}{2 \pi i} \sum_{k=1}^{r}\left\langle D^{(k-1)} f_{k}(t), \frac{1}{t-z}\right\rangle \\
& =\frac{1}{2 \pi i}\left\langle\sum_{k=1}^{r} D^{(k-1)} f_{k}(t), \frac{1}{t-z}\right\rangle .
\end{aligned}
$$

Let

$$
f(x)=\sum_{k=1}^{r} D^{(k-1)} f_{k}(t)
$$

where $f(x) \in D_{L^{p}}^{\prime}$, which implies that $F(z)$ is one of the analytic representations of $f(x)$. 


\subsection{Proof of Corollary 2.3}

In view of the structure formula (see [9])

$$
\begin{aligned}
F(z) & =\frac{1}{2 \pi i}\left\langle f(t), \frac{1}{(t-z)^{j}}\right\rangle \\
& =\frac{1}{2 \pi i} \sum_{k=1}^{r} \int_{\mathbb{R}} f_{k}(t)\left(-\frac{\partial}{\partial t}\right)^{(k-1)}\left(\frac{1}{(t-z)^{j}}\right) d t \\
& =\frac{1}{2 \pi i} \sum_{k=1}^{r} \int_{\mathbb{R}} f_{k}(t) \frac{(k+j-2) !}{(j-1) !(t-z)^{k+j-1}} d t .
\end{aligned}
$$

Similar to the proof of Theorem 2.1, we can see the corollary holds.

\section{Conclusions}

In this paper, we not only obtained the representation of Schrödingerean harmonic functions but also gave a sufficient and necessary condition between the Schrödingerean distributional function and its derivative in the Schrödingerean Hardy space.

\section{Acknowledgements}

We would like to thank the editor, the associate editor and the anonymous referees for their careful reading and constructive comments which have helped us to significantly improve the presentation of the paper. This work was supported by the Natural Science Foundation of Heilongjiang Province (No. A2016209040).

\section{Competing interests}

The authors declare that they have no competing interests.

\section{Authors' contributions}

All authors participated in every phase of research conducted for this paper. All authors read and approved the final manuscript.

\section{Author details}

${ }^{1}$ College of Power and Energy Engineering, Harbin Engineering University, Harbin, 150001, China. ${ }^{2}$ Department of Computer Science and Technology, Harbin Engineering University, Harbin, 150001, China.

\section{Publisher's Note}

Springer Nature remains neutral with regard to jurisdictional claims in published maps and institutional affiliations.

Received: 22 September 2017 Accepted: 28 November 2017 Published online: 13 December 2017

\section{References}

1. Jiang, Z: Some Schrödinger type inequalities for stabilization of discrete linear systems associated with the stationary Schrödinger operator. J. Inequal. Appl. 2016, Article ID 247 (2016)

2. Orton, M: Hilbert transform, Plemelj relation, and Fourier transform of distributions. SIAM J. Math. Anal. 4(4), 656-670 (1973)

3. Jiang, Z, Uso, F: Boundary behaviors for linear systems of subsolutions of the stationary Schrödinger equation. J. Inequal. Appl. 2016, Article ID 233 (2016)

4. Huang, J: A new type of minimal thinness with respect to the stationary Schrödinger operator and its applications. Monatshefte Math. 183(3), 495-507 (2017)

5. Huang, J, Ychussie, B: The modification of Poisson-Sch integral on cones and its applications. Filomat 30(12), 3291-3302 (2016)

6. Lü, F, Ülker, T: Existences of weak solutions for two point boundary value problems of Schrödingerean predator-prey system ant their applications. Adv. Differ. Equ. 2017, Article ID 159 (2017)

7. Pandey, J: The Hilbert Transform of Schwartz Distributions and Applications. Wiley, New York (1996)

8. Priestley, P: Introduction to Complex Analysis. Oxford University Press, Oxford (2003)

9. Schwartz, L: Théorie des distributions. Hermann, Paris (1978) 